containing the embryos immersed in culture medium is then seated in the opening $(F)$. When the 'Lucite' cover $(A)$ is replaced, the pin $\left(D_{2}\right)$ releases the safety latch $\left(D_{2}\right)$ so that the slide $(E)$ can now be moved freely. For exposure of the cells, the slide $(E)$ is pulled out as far as possible, and the lever $\left(H_{1}, H_{2}\right)$ is depressed immediately to push the plaque against the bottom of the exposure dish. At the end of the exposure, the lever is released and the slide is pushed back. The movements can be synchronized so that the duration of exposure can be very accurately regulated. The ejecting lever $(J)$ facilitates removal of the plaque.

The principal features which contribute to the protection of the operator are the 'Lucite' shield $(A)$, the lead-aluminium lid $(C)$, the 'Lucite' enclosure', in which the plaque is transferred to and from the exposure chamber, and the safety latch $(D)$, which holds the radioactive plaque in a sefety position under the lid $(C)$. The lid $(C)$ over the plaque receptacle $(B)$ consists of a piece of lead between two sheets of aluminium-the aluminium stops the beta-particles and, being of low atomic number, generates few Bremsstrahlen, which are easily absorbed by the lead.

Following irradiation, the embryos can be transferred to cover slips in hanging drops of culture medium and sealed over depression slides for microscopic study of the neuroblasts. With radiation-induced mitotic inhibition serving as criterion of effect, the method has given satisfactory results in that all cells treated have been uniformly affected. A detailed. report of the results will appear elsewhere.

This investigation was performed under Contract \# W-7405-Eng-26 for the Atomic Energy Commission at Oak Ridge. We wish to thank Mr. E. B. Darden, jun., for technical assistance and Mr. A. E. Strauchman for drawing the apparatus. [Aug. 2.

' Bizzell, O. M., Burnett, W. T., jun., Tompkins, P. C., and Wish, L., Nucleonic8, 8, 17 (1951).

Sheppard, C. W., and Abele, R. K., AECU, 655 (1949).

'Carlson, J. G., Biol. Bull., 90, 109 (1946).

\section{SELECTIVE PHYTOTOXICITY OF SYNTHETIC GROWTH REGULATORS IN WHEAT, OATS AND BARLEY}

\footnotetext{
HE initial phase of the development of $2: 4$ dichlorophenoxyacetic acid (DCPA or 2,4-D) and 2-methyl-4-chlorophenoxyacetic acid (MCPA) as selective herbicides took place in England between 1943 and 1945. Because of the limitations of wartime, the experiments were confined to the testing of simple inorganic salts, coupled with methods of application which were within the capacities and performance of existing spraying machinery. In consequence, the first recommendations for the use of these synthetic growth regulators in cereal crops centred around the sodium salts applied as a relatively coarse spray of some 100 gallons of aqueous solution per acre.

Within two years cereals were being extensively sprayed in Great Britain and elsewhere. In the United States and Canada, shortage of water on the farm was often a major factor in hindering the more extended use of $2: 4$ dichlorophenoxyacetic acid, and the necessity to economize water led to the development of inexpensive machines capable of applying a
}

few gallons of spray solution per acre. This 'lowvolume' method of application demanded in turn formulations which could be applied in relatively high concentrations, such as the readily soluble ethanolamine salts or esters, applied in oil-water emulsions.

With these twin developments, there came a further major expansion in the acreage treated, first in America and then from 1948 onwards in Great Britain. The change-over from high-volume to low-volume spraying was so rapid that in all countries practice completely outstripped principle, since in the interval there had not been time enough to establish how far the phytotoxic properties of amines and esters differed from those of the original sodium salts, or whether the differences in the mean droplet size associated with varying the volume of application brought about differential effects.

To the research worker it is not, therefore, surprising that with the extended use of the newer techniques and formulations there has been some variability of results. Perhaps the most significant feature is the liability of the cereal crop to be injured, the damage showing itself most clearly in the abnormal inflorescences produced. It was against this background that a symposium on "The Selective Phytotoxicity of 2,4-D and MCPA in Wheat, Oats and Barley" was arranged by the Crop Protection Panel of the Agricultural Group of the Society of Chemical Industry and held on November 8 at the Royal College of Science.

Dr. W. E. Ripper (Pest Control, Ltd.) discussed the results of recent experiments relating to the effects of $2: 4$ dichlorophenoxyacetic acid and 2-methyl-4chlorophenoxyacetic acid on a susceptible dicotyledonous species, namely, mustard. When the sodium salts are applied, formative effects such as epinasty appear first in plants treated with the latter. If rain falls shortly after treatment, much less injury occurs in the plants receiving the former. The replacement of sodium by an amine base leads to some increase in toxicity, but the differences are not very great; neither does the effect of rainfall vary greatly with these changes. When spray droplets on the leaves are allowed to dry out, examination under the microscope shows that crystals of the sodium salt of the dichloro compound are formed much sooner than those of the sodium salt of 2-methyl-4-chlorophenoxyacetic acid, while in the case of the amines at normal air humidities crystallization rarely occurs.

Mr. K. Carpenter (May and Baker, Ltd.) emphasized that in a mixed weed population competition between species is a factor which must be taken into account in interpreting the effects of any spray treatment on the individual species. In the mixed weed flora investigated, the ethyl ester of $2: 4$ dichlorophenoxy. acetic acid applied in an oil emulsion was in general more toxic than aqueous sprays of the trimethylamine salt, but the differences were dependent on the species.

In Europe, Great Britain and America, a wide range of formulations of $2: 4$ dichlorophenoxyacetic acid has been employed in investigations relating to cereals, while 2-methyl-4-chlorophenoxyacetic acid has been tested almost entirely as the sodium salt. Therefore, an assessment of their comparative effects based on the same type of formulation is often not possible, while the further difficulty arises that in different countries the relatjve interest in these two herbicides has greatly varied. These difficulties were pointed out by Dr. H. P. Allen (Plant Protection, Ltd.) in his general review of the direct effects of these synthetic growth regulators on cereal crops. 
There is evidence that cereal crops differ in their liability to injury; oats appear to be the most susceptible, barley is intermediate and winter wheat the most resistant. Dr. Allen's experiments on barley have indicated that with a change-over from the sodium salts to the $n$-butyl esters, the greater toxicity to the crop of $2: 4$ dichlorophenoxyacetic acid is accentuated. Mr. J. Fryer (Agricultural Research Council Unit of Experimental Agronomy, Oxford) stressed that any attempt to define the effects on cereals of formulation and volume of application demands an extensive field programme covering several years. A preliminary examination of the results for the 1951 trials on barley suggests that the degree of injury brought about by the application of the triethanolamine salt of $2: 4$-dichlorophenoxyacetic acid at the rate of $0.5 \mathrm{lb}$. of the acid per acre is roughly equivalent to the effects produced by the sodium salt of 2-methyl4-chlorophenoxyacetic acid at $2 \mathrm{lb}$. per acre. Spraying at an early stage prior to the formation of tillers produces the largest number of abnormalities; but even when the proportion of abnormal ears is exceptionally high, this does not necessarily lead to any appreciable loss of yield, although the quality of the grain may be affected.

K. Holly (Agricultural Research Council Unit of Experimental Agronomy, Oxford) pointed out that in commercial practice it is commonly held that a specific amount of these two herbicides is required per acre to destroy a particular weed species and that the volume of solution applied is of little importance. Field investigations at Oxford have revealed a great variability in the effects of changing volume-rate on different species and between different experiments. From experiments in the greenhouse it is already evident that the amount of spray solution retained by the shoot is dependent on the volume of solution applied, and that with the test plants used (mustard and barley) there were marked specific differences in retention as the volume changed. Since a change of droplet size could also affect penetration into the leaf and since the effects of decreasing droplet size on penetration tended to be the reverse of those on retention, it was not surprising that in the field variable results were obtained. Dr. E. K. Woodford (Agricultural Research Council Unit of Experimental Agronomy, Oxford) stated that in his opinion the greater toxicity of the dichloro compound in the field cannot be satisfactorily accounted for in terms of retention and penetration. On the other hand, the evidence, although surprisingly scanty, indicates that at cell-level there is little difference between the two compounds in their inherent toxicity. One is therefore left with the conclusion that the major difference between the compounds is a question of transport or persistence within the plant.

Lastly, Prof. L. J. Audus (Bedford College, London) dealt with some of the factors relating to the breakdown by micro-organisms of $2: 4$ dichlorophenoxyacetic acid, 2-methyl-4-chlorophenoxyacetic acid and $2: 4: 5$-trichlorophenoxyacetic acid in the soil. In perfusion experiments there is first a lag phase when no breakdown occurs followed by a rapid rate of decomposition. The lag phase is shortest for the dichloro compound; complete breakdown of the trichloro compound has not been found even after a pericid of months. Once the phase of rapid decomposition has been reached, there is no subsequent lag perir $\mathbf{d}$ when further additions of the dichloro or methyl compound are made. Since the decomposition can be inhibited by the addition of sodium azide, it is clearly due to micro-organisms; in fact, an organism belonging to the B. globiformis group can be readily isolated. So far attempts to grow this organism in culture media have not been successful, and the further elucidation of the mechanism of breakdown is thereby being hindered.

G. E. BLACKMAN

\section{INSTITUTION OF GAS ENGINEERS AUTUMN RESEARCH MEETING}

THE seventeenth autumn research meeting of the Institution of Gas Engineers was held in London during November 27-28, 1951, under the presidency of Mr. J. H. Dyde.

A statement by Colonel H. C. Smith, then deputy chairman of the Gas Council, which opened the proceedings, outlined the progress which had been made with regard to the future organization of research in the gas industry. The Gas Council considered that its responsibility was primarily fur fundamental research, and took the view that the gas industry's existing research body, the Gas Research Board, was not an appropriate machine for satisfying the duties imposed upon the Council by the Gas Act of 1948. Accordingly, a research advisury committee was appointed which, after reviewing existing investigations, recommended the establishment. of research stations. Two such stations have now been formed, in London and Birmingham, under the direction of Dr. H. Hollings and Dr. F. J. Dent, respectively. In addition, continued Colonel Smith, the connexion between the gas industry and the University of Leeds is to be maintained through a joint committee of the University and the Gas Council. Finally, a small research liaison committee has been formed to ensure effective collaboration and co-ordination of the work.

A feature common to several of the technical papers presented later during the meeting was the successful application of new scientific techniques to the current problems of the gas industry. Thus, C. H. Lewis described a methed of measuring coal travel in continuous vertical retorts which has the advantage over previous methcds of being non-destructive, and hence applicable at any stage in the working life of a retort. A tally of radioactive cobalt is intruduced into the coal charge at the top of the retort, and its position is determined at intervals during its descent by means of water-cooled Geiger counters placed in the combustion chambers.

Again, the high paramagnetic susceptibility of oxygen has been used as the basis of an instrument designed to record the oxygen content of a gas in the range 0-2 per cent by volume. A description of the instrument, which depends for its operation on the generation of a magnetic wind, was given by $R$. $H$. Griffith, who also presented some results obtained by its use for the automatic control of a catalytic plant for the removal of organic sulphur compounds from coal gas.

In the field of gas utilization, a better control of combustion processes, and improved heat transfer from flame gases, should follow from the wider application of experimental techniques adapted by W. A. Simmonds and M. J. G. Wilson for following the path of secondary air towards and into flames. Both the quartz-fibre anemometer and the powder method of flow visualization have been used for determining the flow of air towards small, radially symmetrical diffusion flames of several inflammable 